\title{
Picrasma quassioides Extract Elevates the Cervical Cancer Cell Apoptosis Through ROS-Mitochondrial Axis Activated p38 MAPK Signaling Pathway
}

\author{
YI-XI GONG ${ }^{1 *}$, YUE LIU ${ }^{1 *}$, YING-HUA JIN ${ }^{2 *}$, MEI-HUA JIN ${ }^{1}$, YING-HAO HAN ${ }^{1}$, JING LI ${ }^{1}$, GUI-NAN SHEN ${ }^{1}$, \\ DAN-PING XIE ${ }^{1}$, CHEN-XI REN ${ }^{1}$, LI-YUN YU ${ }^{1}$, DONG-SEOK LEE ${ }^{3}$, JI-SU KIM ${ }^{4}$, YU-JIN JO ${ }^{4}$, \\ JEONGWOO KWON ${ }^{5}$, JAIHYUNG LEE ${ }^{6}$, YANG HO PARK ${ }^{7}$, TAEHO KWON ${ }^{4}$, YU-DONG CUI ${ }^{1}$ and HU-NAN SUN ${ }^{1}$ \\ ${ }^{I}$ College of Life Science and Technology, Heilongjiang Bayi Agricultural University, \\ Heilongjiang, Daqing, P.R. China; \\ ${ }^{2}$ Library and Information Center, Heilongjiang Bayi Agricultural University, Daqing, P.R. China; \\ ${ }^{3}$ School of Life Sciences, KNU Creative BioResearch Group (BK21 plus project), \\ Kyungpook National University, Daegu, Republic of Korea; \\ ${ }^{4}$ Primate Resources Center, Korea Research Institute of Bioscience \\ and Biotechnology (KRIBB), Jeonbuk, Republic of Korea; \\ ${ }^{5}$ Department of Animal Sciences, Chungbuk Natonal University, Cheongju, Republic of Korea; \\ ${ }^{6}$ Haeam Convalescence Hospital, Gyeonggi, Republic of Korea; \\ ${ }^{7}$ Park Yang Ho BRM Institute, Seoul, Republic of Korea
}

\begin{abstract}
Background/Aim: Picrasma quassioides (P. quassioides) is used in traditional Asian medicine widely for the treatment of anemopyretic cold, eczema, nausea, loss of appetite, diabetes mellitus, hypertension etc. In this study we aimed to understand the effect of P. quassioides ethanol extract on SiHa cervical cancer cell apoptosis. Materials and Methods: The P. quassioides extract-induced apoptosis was analyzed using the MTT assay, fluorescence microscopy, flow cytometry and western blotting. Results: P. quassioides extract induced cellular apoptosis by increasing the accumulation of cellular and mitochondrial reactive oxygen species (ROS) levels and inhibiting ATP synthesis.
\end{abstract}

This article is freely accessible online.

*These Authors contributed equally to this work.

Correspondence to: Taeho Kwon, Primate Resources Center, Korea Research Institute of Bioscience and Biotechnology (KRIBB), 35133 Neongme-gil, Ibam-myeon, Jeongeup-si, Jeonbuk, 56216, Republic of Korea. Tel: +82 635705316, Fax: +82635705309 , email: kwon@kribb.re.kr and Yu-Dong Cui and Hu-Nan Sun, College of Life Science \& Technology, Heilongjiang Bayi Agricultural University, Xinyanglu, 163319, Daqing, P.R. China. Tel: +86 4596819300, Fax: +86 4596819295, e-mail: cuiyudong@126.com and sunhunan76@163.com

Key Words: Picrasma quassioides, cervical cancer, apoptosis, ROS, mitochondria.
Pretreatment with $N$-Acetylcysteine (NAC), a classic antioxidant, decreased the intracellular ROS production and inhibited apoptosis. In addition, the P38 MAPK signaling pathway is a key in the apoptosis of SiHa cells induced by the P. quassioides extract. Conclusion: The P. quassioides extract exerts its anti-cancer properties on SiHa cells through ROS-mitochondria axis and P38 MAPK signaling. Our data provide a new insight for P. quassioides as a therapeutic strategy for cervical cancer treatment.

Cervical cancer is the fourth most common cancer affecting women worldwide (1), putting a huge financial and medical burden on society, so it is necessary to work together globally to improve its treatment. At present, the methods of treating cervical cancer mainly include surgery, chemotherapy and adjuvant chemotherapy $(2,3)$, with certain side effects, such as loss of appetite and weight loss. Screening of natural products from plant sources, such as herb extracts, with minimal side effects that can be obtained in a cost-effective manner have received global attention for treating several types of cancers (4-6).

Picrasma quassioides ( $P$. quassioides) is a Asian traditional medicine mainly distributed in Korea and South China (7). The bark, roots, stems and leaves are traditionally used for the treatment of anemopyretic cold, sore throat, dysentery, eczema, nausea, loss of appetite, diabetes mellitus, hypertension and other conditions (8-10). Chemical investigation has shown that alkaloids, including $\beta$-carboline $(11,12)$, canthinone $(13)$ and bis $\beta$-carboline alkaloids $(14$, 
15) are the principal active components in $P$. quassioides. These active materials show anti-inflammatory, antihypertensive and anti-angiogenic properties in humans $(8,9)$. At the same time, recent studies have shown that the monomers extracted from P. quassioides have the ability to increase intracellular reactive oxygen species and induce apoptosis in HepG2 and Hep3B liver cancer cells $(16,17)$. Despite these data, the anti-cancer effect of $P$. quassioides on cervical cancer and the possible underlying mechanism has not yet been studied.

Reactive oxygen species (ROS) formed from various cellular metabolites play a vital role in many biological processes, including oxygen sensing, cell differentiation and adaptive immunity (18). Disturbances in the balance between intracellular antioxidants and ROS can lead to the dysfunction of mitochondria (19), which can promote cell and tissue oxidative stress in vivo and in vitro. Most active radicals are considered as natural by-products of mitochondrial activation. Generally, low levels of ROS can serve as signal molecules to regulate numerous physiological processes, while excess levels can lead to caspase activation and cytochrome c release and promote cell death (20), causing various diseases, such as cardiovascular disease, diabetes, and cancer (21, 22). Compared to normal cells, cancer cells have higher ROS levels. High levels of ROS change the redox state of the cell itself, promoting the occurrence and development of malignant phenotypes as reflected in the continuous proliferation of cancer cells (20).

The biochemical characteristics of cancer cells make them more vulnerable to further ROS increase induced by exogenous ROS-generating agents (pro-oxidants) compared to normal cells (23). Based on these facts, new anti-cancer strategies for ROS have been developed to selectively kill cancer cells by promoting excessive ROS production and inducing cancer cell mitochondrial dysfunction (24-26).

Herein, the effect of $P$. quassioides extraction on the survival of cervical cancer was evaluated by examining the viability, apoptosis and the changes in apoptosis-related protein expression in $\mathrm{SiHa}$ cervical cancer cells. We also investigated the effect of $P$. quassioides extract on $\mathrm{SiHa}$ cell migration, colony formation, mitochondrial membrane potential, cellular and mitochondrial ROS levels and ROSdependent signaling pathways, such as MAPK signaling. Our findings may give a new sight in understanding the potential anti-cancer properties of $P$. quassioides extract on cervical cancer.

\section{Materials and Methods}

Extract preparation. A crude extract of Picrasma quassioides was prepared essentially as described before (27). Briefly, air-dried stems of Picrasma quassioides $(50 \mathrm{~g}$ ) were soaked in $500 \mathrm{ml}$ of ethyl alcohol, and then extracted by shaking it at $200 \mathrm{rpm}$ for $5 \mathrm{~h}$ at $37^{\circ} \mathrm{C}$. The filtrate was recovered, and an additional $500 \mathrm{ml}$ of ethyl alcohol were used for filtering the residue. The process of recovery and filtration of the extract was repeated three times and it was concentrated on a rotary evaporator for $12 \mathrm{~h}$ at $55^{\circ} \mathrm{C}$. The concentrated extract was labeled as $\mathrm{PQ}$ and stored in a refrigerator at $4^{\circ} \mathrm{C}$ until further use.

Cell culture. SiHa cervical cancer and QSG-7701 hepatocyte normal cells were cultured in DMEM (Invitrogen, Carlsbad, CA, USA) medium supplemented with $10 \%$ fetal bovine serum (FBS, Hyclone, Logan, UT, USA), penicillin (100 U/ml) and streptomycin $(100 \mathrm{mg} / \mathrm{ml})(\mathrm{P} / \mathrm{S})$ (Solarbio life sciences, Beijing, PR China), and were incubated at $37^{\circ} \mathrm{C}$ with $5 \% \mathrm{CO}_{2}$.

Cell viability assay. The cell viability was analyzed using MTT [3(4,5-dimethylthiazol-2-yl)-2,5 -diphenyltetrazolium bromide, Sigma-Aldrich, St. Louis, MO, USA] assay and CCK-8 [2-(2methoxy-4-nitrophenyl)-3-(4-nitrophenyl) -5-(2,4-isulfophenyl)-2Htetrazolium, monosodium salt, cell counting Kit-8, MedChem Express, Shanghai, PR China]. CCK-8 is more sensitive than MTT, but there is no significant difference between them in detecting cell viability. The SiHa and QSG-7701 cells were seeded in 96-well plates at $4 \times 10^{3}$ cells/well and treated with PQ for $24 \mathrm{~h}$. Then, $10 \mu \mathrm{l}$ $(0.5 \mathrm{mg} / \mathrm{ml})$ of MTT or $10 \mu \mathrm{l}$ of the CCK-8 were added to each well and incubated for $2 \mathrm{~h}$ at $37^{\circ} \mathrm{C}$ in $5 \% \mathrm{CO}_{2}$. Subsequently, the supernatant was removed and formazan was solubilized with dimethyl sulfoxide. Absorbance was measured at $490 \mathrm{~nm}$ or $450 \mathrm{~nm}$ using a UV MAX kinetic microplate reader (Molecular Devices, LLC, Sunnyvale, CA, USA).

Apoptosis detection by Annexin V-FITC. The SiHa cells were seeded in 6-well plates at $2 \times 10^{5}$ cells/well following a $24 \mathrm{~h}$ incubation with PQ treatment. To detect apoptosis, cells were prepared using the Annexin V-FITC and propidium iodide (PI) detection Kit (Solarbio life sciences, Beijing, PR China), according to the manufacturer's protocol and were analyzed by fluorescence microscopy (EVOS ${ }^{\circledR} \mathrm{xl}$ core cell culture microscope, Advanced Microscopy Group, Paisley, Scotland, UK) and flow cytometry (FACSCalibur, BD Biosciences, Franklin Lakes, NJ, USA). The results were analyzed using the WinMDI (Version 2.9, BD Biosciences) software.

Figure 1. Effect of $P Q$ on cell viability, apoptosis, cell migration and colony formation in SiHa cells. (A) Cell viability of SiHa cervical cancer cells and QSG-7701 normal liver cells were measured by MTT assay following treatment with $P Q(0,20,30,40,50,60 \mu \mathrm{g} / \mathrm{ml})$ and 5$F U(60 \mu \mathrm{g} / \mathrm{ml}) .5-F U$ caused a decrease in cell viability in both SiHa and QSG-7701 cells, whereas $P Q$ only affected the viability of $\mathrm{SiHa}$ cancer cells. (B) Annexin V-FITC/PI staining and flow cytometry (left side graphs) and increased levels of SiHa cells apoptosis after $P Q$ treatment quantified (right side graph). (C) Fluorescence and light microscopy images of SiHa cells stained with Annexin-V and PI showed increased apoptosis and levels of dead cells with increasing concentration of $P Q$, respectively. (D) Wound healing assay (left panel) and colony formation assay (right panel) of SiHa cells showing their decreased migration capacity after $P Q$ treatment (scale bar $=100 \mu M$ ). Data are presented as the mean \pm standard error of the mean from three different samples. ${ }^{*} p<0.05, * * p<0.01, * * * p<0.001$ 
A

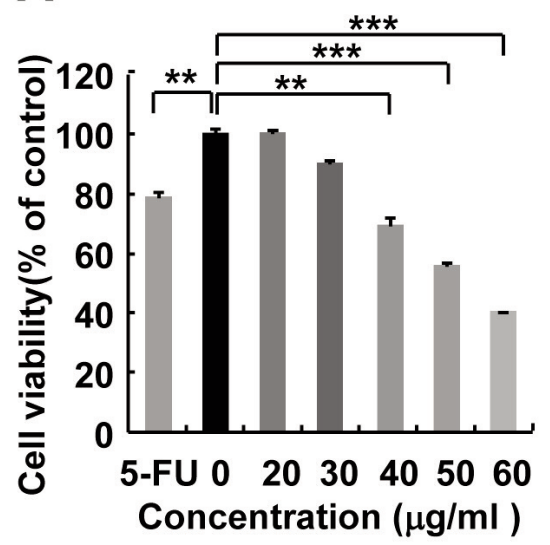

B

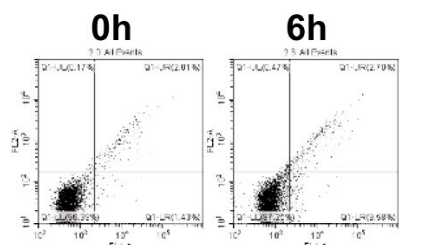

$12 \mathrm{~h}$

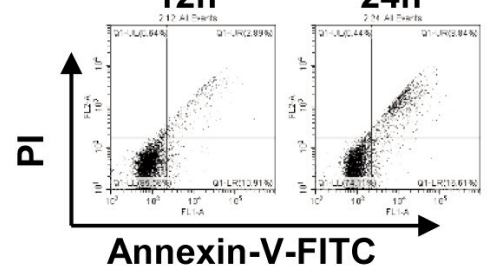

C

C $\mu \mathrm{g} / \mathrm{ml}$

$40 \mu \mathrm{g} / \mathrm{ml}$

$60 \mu \mathrm{g} / \mathrm{ml}$

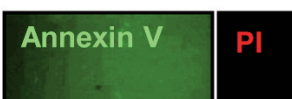

Oh

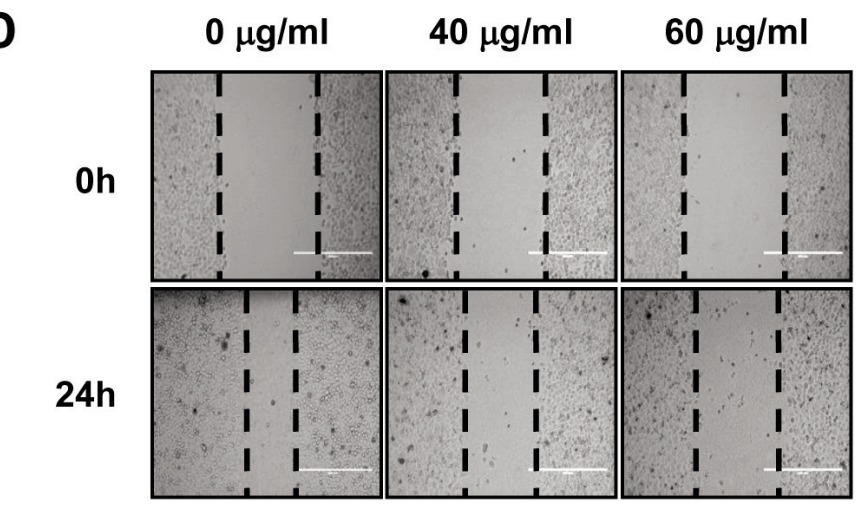

$$
\text { I. }
$$
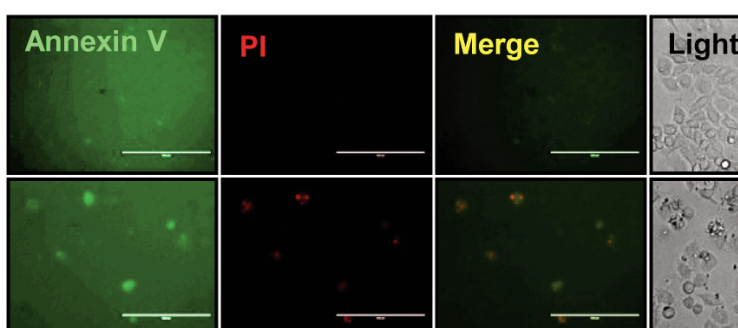

QSG-7701

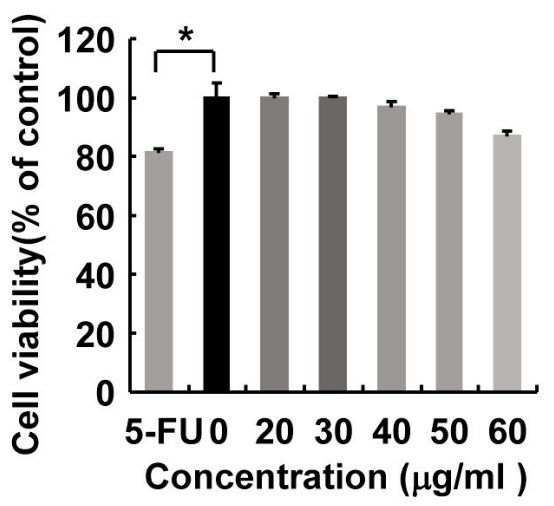

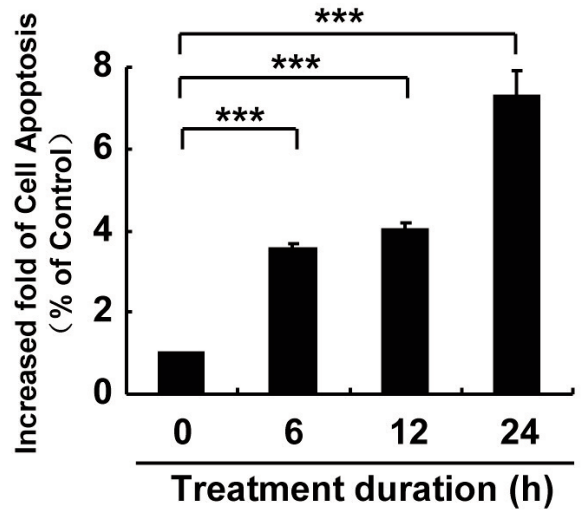


A

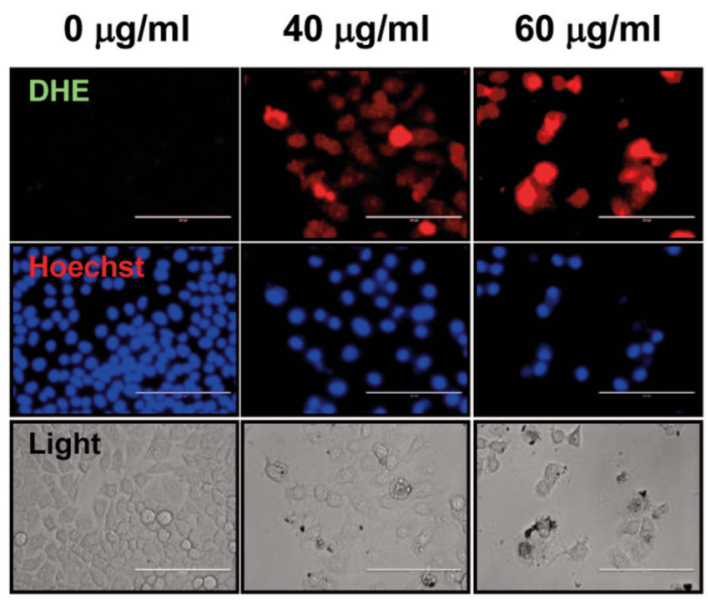

C

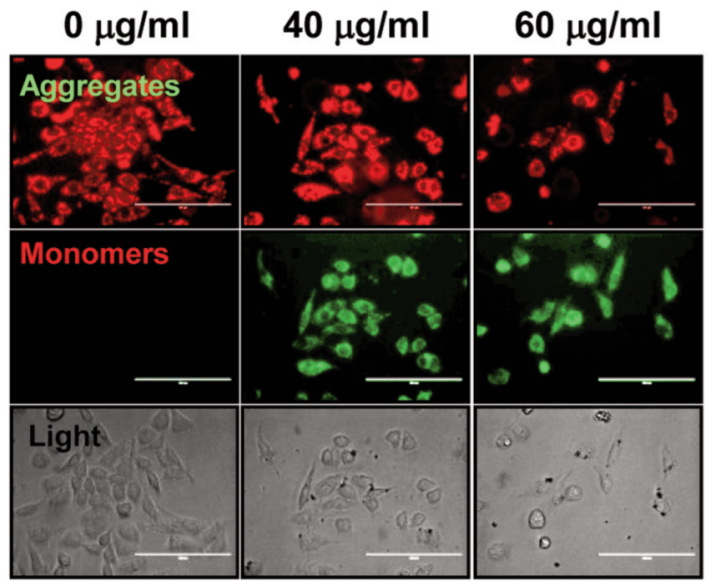

B

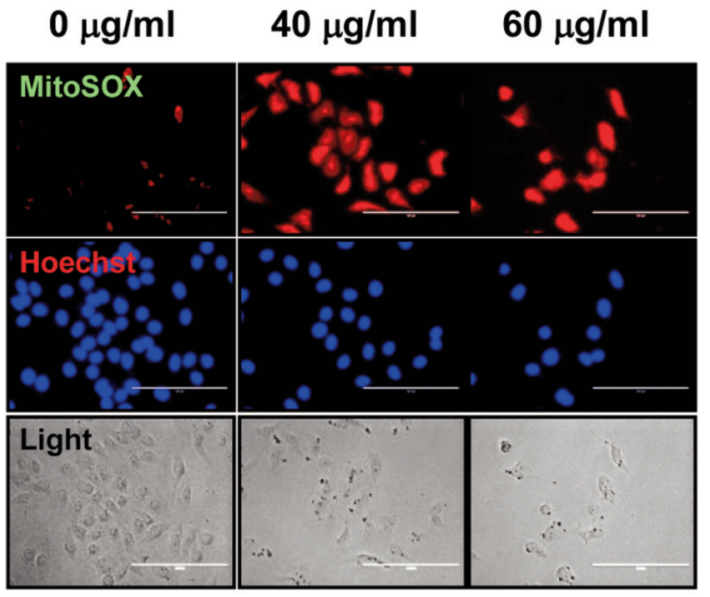

D

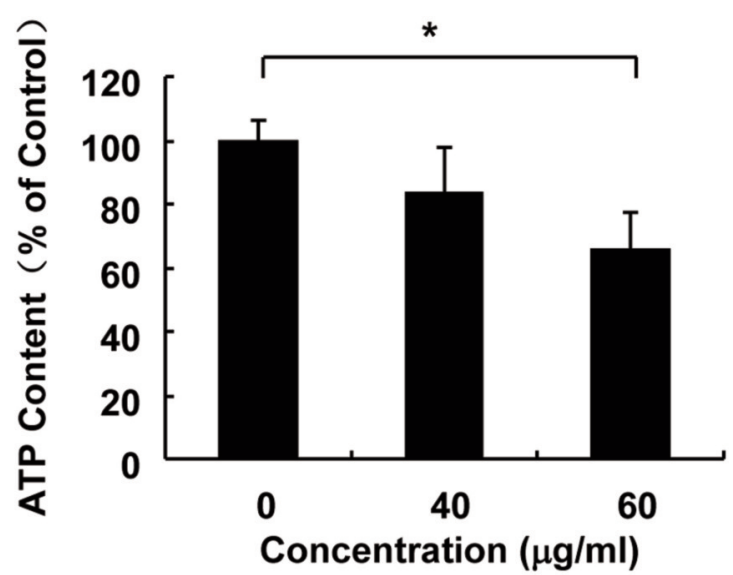

Figure 2. Effect of $P Q$ on cellular and mitochondrial ROS, mitochondrial membrane permeability and ATP content in SiHa cells. (A) Dihydroethidium (DHE) and Hoechst staining of SiHa cells after treatment with increasing concentrations of $P Q$ showed increased cellular reactive oxygen species (ROS) levels, (B) MitoSOX and Hoechs staining show increased mitochondrial ROS in cervical cancer cells, and (C) increased JC-1 staining showed an increase in the mitochondrial membrane potential. (D) The ATP levels in SiHa cells decreased following PQ treatment. Data are presented as the mean standard error of the mean of three different samples. ${ }^{*} p<0.05$.

Wound healing assay. SiHa cells were seeded in 6-well plates at $1 \times 10^{6} \mathrm{cells} /$ well. A linear scratch was made by introducing a small pipette tip in the cell monolayer. The cells were rinsed with $1 \times \mathrm{PBS}$ three times and PQ $(60 \mu \mathrm{g} / \mathrm{ml})$ was added. Photomicrographs of the migrated cells were taken at 0 and $24 \mathrm{~h}$ using a fluorescence microscope (EVOS ${ }^{\circledR} \mathrm{xl}$ core cell culture microscope).

Colony-formation assay. SiHa cells $\left(1 \times 10^{3}\right.$ cells/well) were seeded in six-well plates, treated with PQ $(60 \mu \mathrm{g} / \mathrm{ml})$ and maintained at $37^{\circ} \mathrm{C}$ in a $5 \% \mathrm{CO}_{2}$ incubator for 10 days. The cells were then washed with $1 \times \mathrm{PBS}$, fixed for 10 min with $3.7 \%$ formaldehyde, treated for $20 \mathrm{~min}$ with methanol, and stained for $30 \mathrm{~min}$ with $0.05 \%$ crystal violet. The plates were washed with $1 \times \mathrm{PBS}$ three times before image capturing.
Detection of ROS production. Cells were seeded in 6-well plates at $2 \times 10^{5}$ cells/well and treated with PQ for $24 \mathrm{~h}$. The changes in cellular and mitochondrial ROS levels were determined using Dihydroethidium (DHE. Beyotime Biotechnology, Shanghai, P.R. China), which is specific for superoxide and hydrogen peroxide in living cells and MitoSOX (Thermo Fisher Scientific, Waltham, MA, USA) staining, which detects superoxide in the mitochondria of living cells. Nuclei were visualized using the Hoechst 32258 (Thermo Fisher Scientific, Waltham, MA, USA) staining. Nuclear staining was observed qualitatively under a microscope after $20 \mathrm{~min}$ of incubation with the Hoechst dye.

Mitochondrial depolarization assay. Changes in the levels of the cell mitochondrial membrane potential in SiHa cells treated with PQ 
A

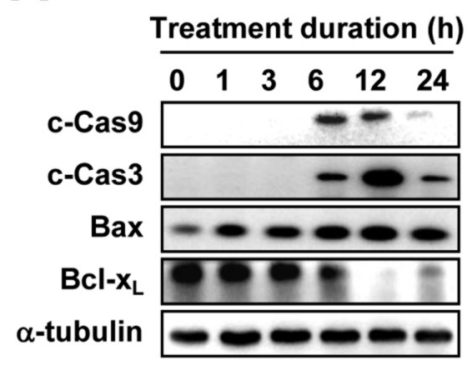

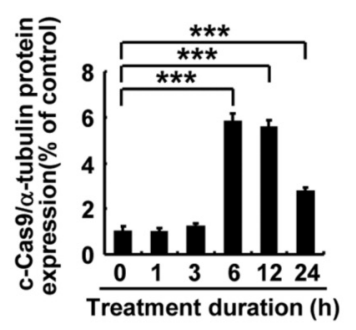

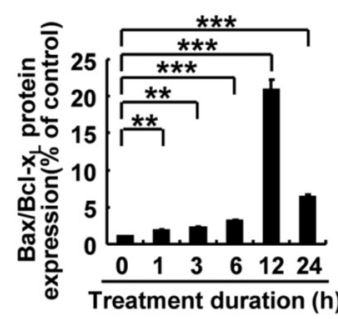

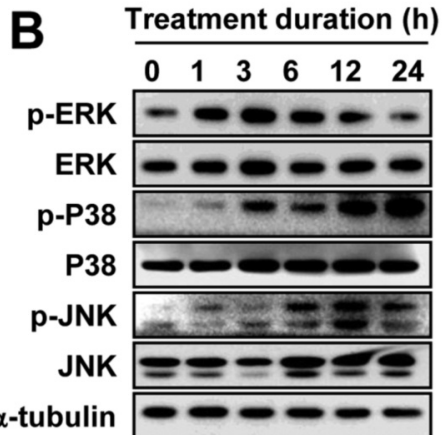

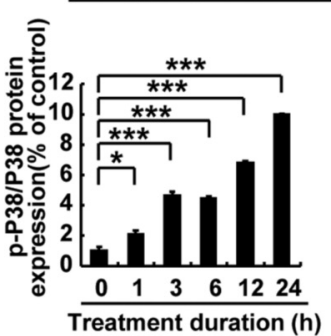

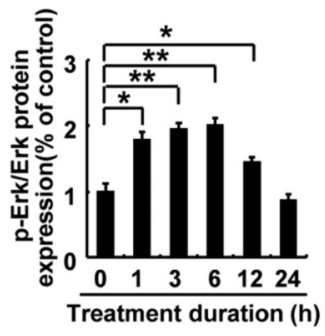

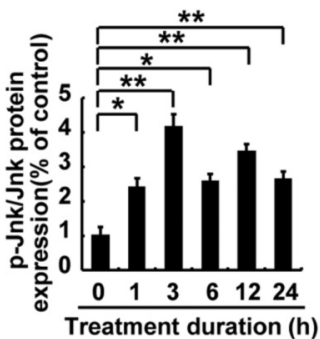

C
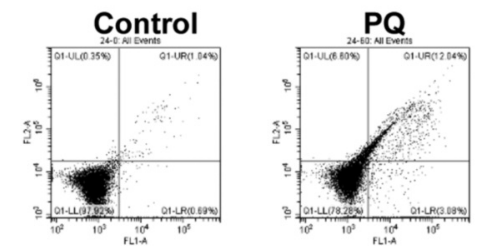

$\mathrm{PD} 98059+\mathrm{PQ}$

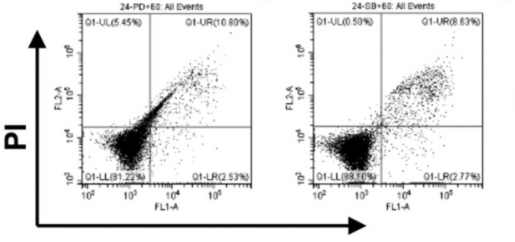

Annexin-V-FITC
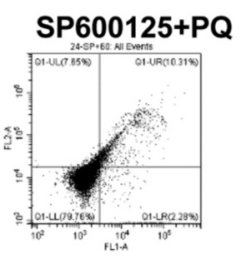

D

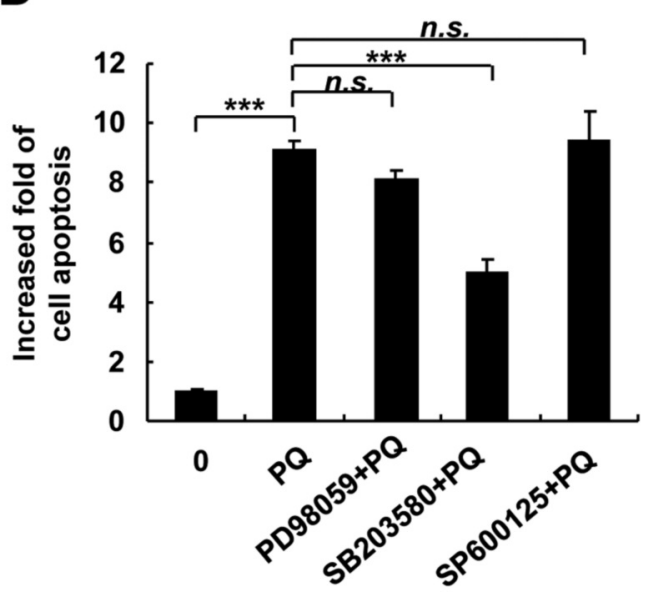

Figure 3. Effect of $P Q$ on the expression of apoptosis and apoptosis-related proteins as well as MAPK signaling pathway activation in SiHa cells. Western blotting shows (A) increased expression of pro-apoptotic c-cas3, c-cas 9, Bax and decreased expression of the anti-apoptotic Bcl- $x_{L}$. (B) $P Q$ treatment also increased the phosphorylated proteins $p-38, E R K$ and JNK, although p-ERK levels returned to normal after a $24 \mathrm{~h}$ incubation with PQ. (C) PQ induces apoptosis in SiHa cells and the use of the SB203580 p38 inhibitor repressed the PQ-induced cellular apoptosis, whereas the inhibitors of ERK (PD98059) and JNK (SP600125) did not. Quantification of these data are presented by the mean $\pm S D$ of $P Q$-treated cells with and without the different inhibitors fromthree different experiments. ${ }^{*} p<0.05, * * p<0.01, * * * p<0.001$.

were determined using JC-1 (Beyotime, Shanghai, PR China). Cells were incubated with $20 \mathrm{mM} \mathrm{JC}-1$ for $15 \mathrm{~min}$ at $37^{\circ} \mathrm{C}$ and then washed with PBS. After washing with PBS, images were taken using a fluorescence core cell culture microscope $\left(\operatorname{EVOS}^{\circledR} \mathrm{x}\right)$ and the fluorescence intensities were observed qualitatively.

Intracellular ATP determination. Cells were seeded in 6-well plates at $2 \times 10^{5}$ cells/well and treated with PQ for $24 \mathrm{~h}$. Adenosine triphosphate (ATP) were determined using the ATP determination Kit (Thermo Fisher Scientific, Waltham, MA, USA). Luminance
(RLU) was immediately measured using a fluorescent chemical analyzer (Infinite 200 PRO Microplate Reader, Tecan Trading AG, Männedorf, Switzerland) according to the manufacturer's protocol.

Western blot analysis. Cell protein lysates were separated in $12 \%$ sodium dodecyl sulfate-polyacrylamide gels and transferred onto nitrocellulose membranes (Millipore, Bedford, MA, USA). The membranes were blotted with primary antibodies against caspase3 (Cell Signaling Technology, Beverly, MA, USA, \#9661), caspase-9 (Cell Signaling Technology, \#9505), Bax (Abcam Cambridge, MA, 
A

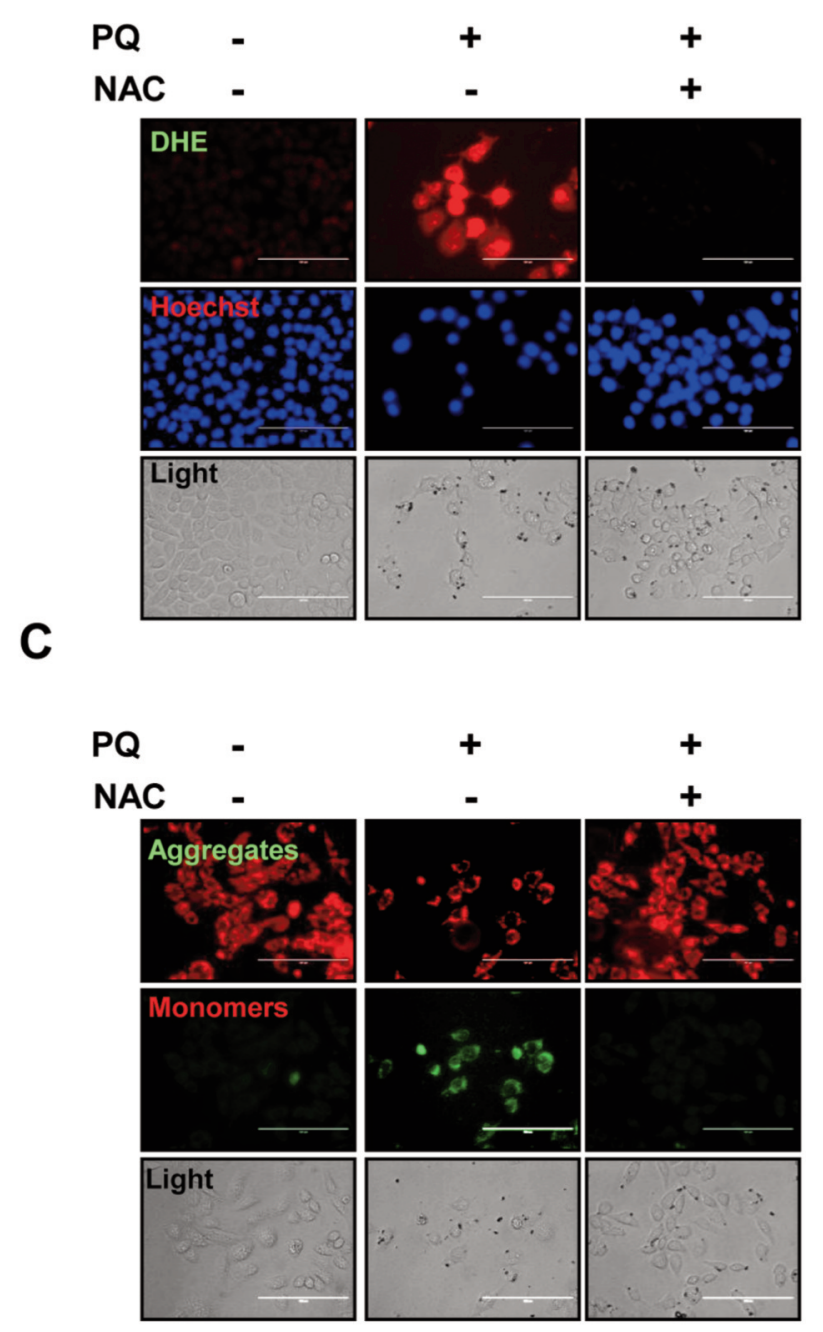

B

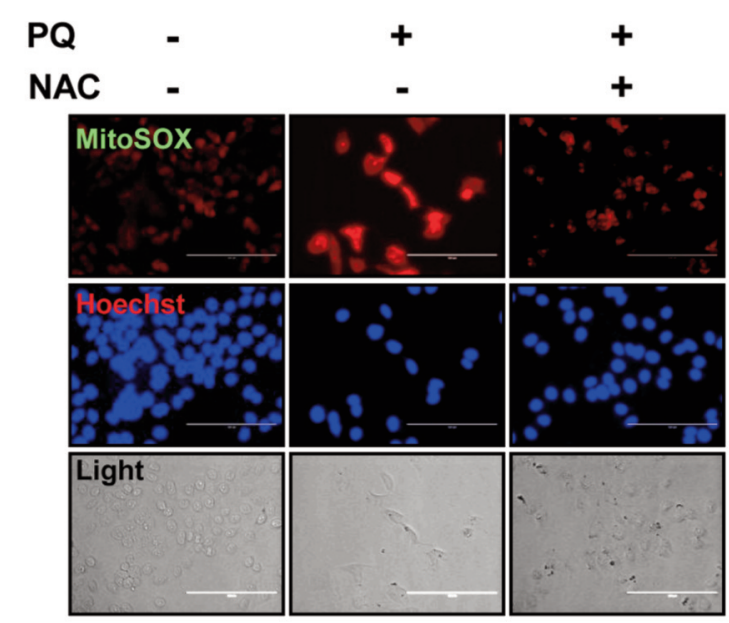

D

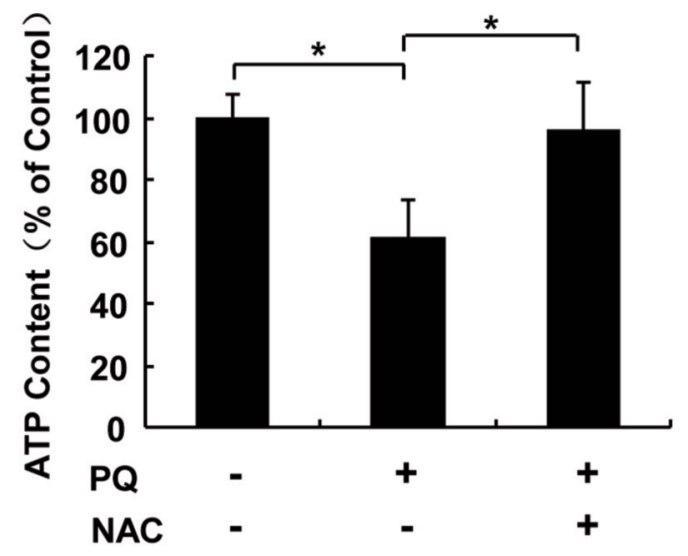

Figure 4. The regulatory function of $P Q$-induced mitochondria dysfunction is dependent on cellular ROS levels. Cell pre-treated with N-Acetylcysteine $(N A C)$ followed by treatment with $P Q$ showed $(A)$ reduced cellular and $(B)$ mitochondrial ROS levels as well as $(C)$ the mitochondrial membrane potential in SiHa cells. (D) On the contrary, NAC treatment significantly restored the PQ-induced inhibition of ATP synthesis. Data are presented as the mean \pm standard error of the mean from three different experiments. * $p<0.05$.

USA, \#32503), Bcl-x $\mathrm{x}_{\mathrm{L}}$ (Santa Cruz Biotechnology, Dallas, TX, USA, \#sc-8392), ERK (Santa Cruz Biotechnology, \#sc-135900), pERK (Santa Cruz Biotechnology, \#sc-7383), P38 (AbFrontier, Seoul, Republic of Korea, \#LF-MA0126), p-P38 (Santa Cruz Biotechnology, \#sc-7973), JNK (Santa Cruz Biotechnology, \#sc7345), p-JNK (Santa Cruz Biotechnology, \#sc-6254), $\alpha$-tubulin (Abcam Cambridge, \#ab7291) (dilution 1:5,000) at $4^{\circ} \mathrm{C}$ overnight. The membranes were washed five times with Tris buffered saline containing Tween-20 (TBST) [10 mM Tris $\mathrm{HCl}(\mathrm{pH} 7.5), 150 \mathrm{mM}$ $\mathrm{NaCl}$ and $0.2 \%$ Tween-20] and were subsequently incubated with Horseradish Peroxidase-conjugated goat anti-rabbit IgG (Sangon Biotech, Shanghai, PR China) or anti-mouse IgG (Sangon Biotech) for $1 \mathrm{~h}$ at room temperature (RT). After the removal of excess antibodies by washing with TBST, specific binding was detected using a chemiluminescence detection system (GE Healthcare Life Sciences, Chalfont, UK), according to the manufacturer's protocol.

Statistical analysis. The data are depicted as mean \pm SEM values. Student's $t$-tests were performed using GraphPad Prism 4.0 software (GraphPad Software Inc., San Diego, CA, USA), and values of $p<0.05$ were considered indicative of significant difference.

\section{Results}

$P Q$ treatment increased the cellular apoptosis, cell migration and colony formation in SiHa cervical cancer cells. To investigate effect of PQ on cellular cytotoxicity, the SiHa 
A

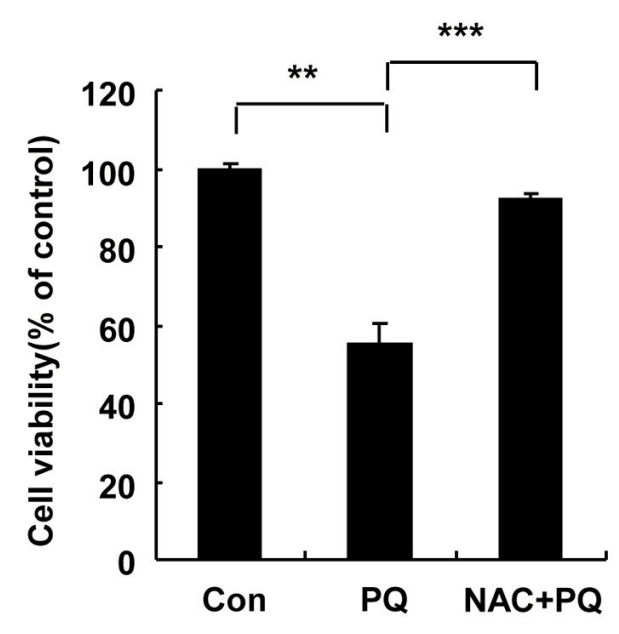

C

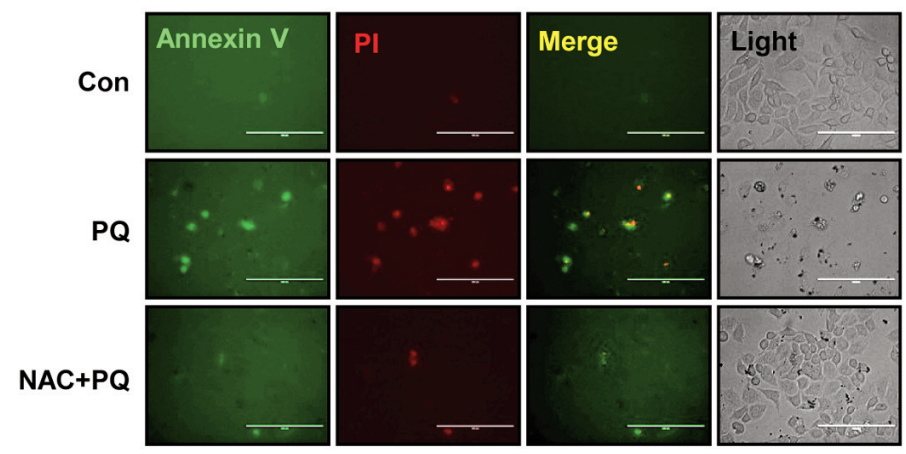

B

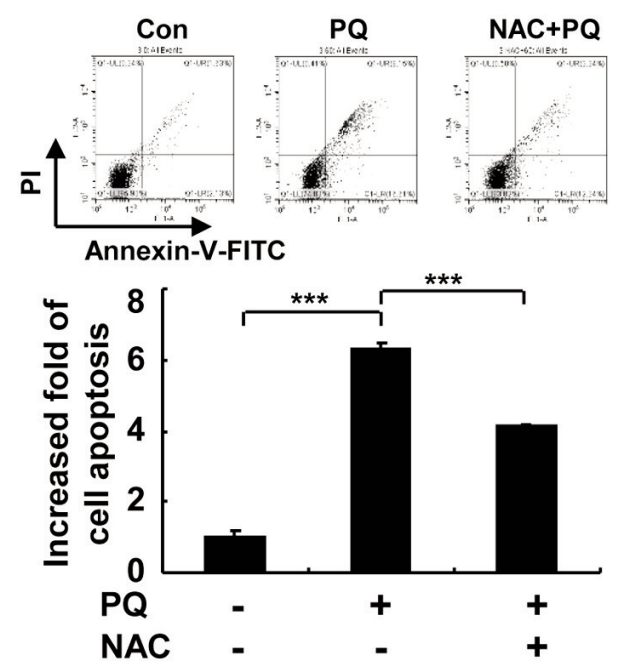

D

$\mathbf{E}$
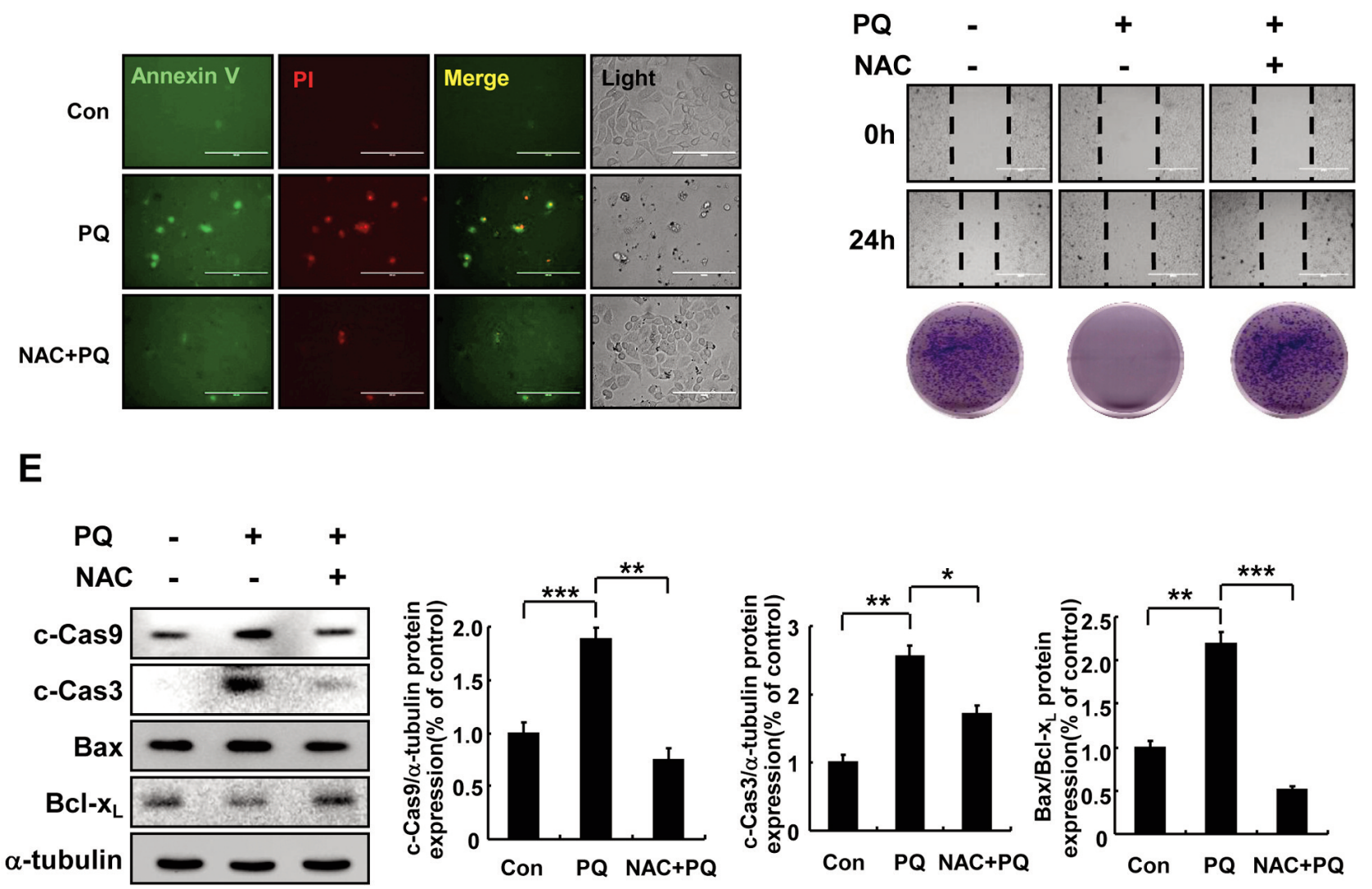

Figure 5. Effect of NAC treatment on cell viability, apoptosis, cell migration, colony formation and apoptosis-related protein expression in SiHa cells. (A) NAC treatment restored the PQ-induced decrease in cell viability, and $(B, C)$ decreased cell apoptosis as shown by flow cytometry and fluorescence microscopy, respectively. (D) Wound healing assay and colony formation assay showed a restoration in the levels of migratory and proliferative ability of $P Q$ - treated cervical cancer cells when these were pre-treated with NAC. (E) NAC pretreatment decreased the expression levels of pro-apoptotic c-cas 3 and c-cas9, as well as the anti-apoptotic Bcl-x $x_{L}$, as detected by Western blot. $(F)$ WB data are presented as the mean \pm standard error of the mean from three different experiments. ${ }^{*} p<0.05, * * p<0.01, * * * p<0.001$. 
cervical cancer and QSG-7701 normal liver cells (used as an experimental control) were treated with 5 -FU $(60 \mu \mathrm{g} / \mathrm{ml})$ and PQ in various concentrations $(0,20,30,40,50$ and $60 \mu \mathrm{g} / \mathrm{ml})$ for $24 \mathrm{~h}$ before the MTT assay. The results showed that both 5FU and PQ have strong cytotoxic effects on SiHa cervical cancer cells with higher levels exhibited by PQ compared to the 5-FU treatment, while both of them have a little cytotoxic effect on QSG-7701 normal liver cells (Figure 1A). To determine whether PQ can induce cell apoptosis, the SiHa cells were treated with PQ $(0,40$ and $60 \mu \mathrm{g} / \mathrm{ml})$ for indicated duration times $(0,6,12$ and $24 \mathrm{~h})$ and were stained using the Annexin$\mathrm{V}$ FITC and PI detection Kit. The apoptotic cells using recombinant Annexin V conjugated to green-fluorescent FITC dye, PI stains necrotic cells with red fluorescence. After treatment with both probes, apoptotic cells show green fluorescence, dead cells show both red and green fluorescence, and live cells show little or no fluorescence. The results revealed that PQ treatment can significantly increase the cellular apoptosis in a time- and dose-dependent manner in $\mathrm{SiHa}$ cervical cancer cells (Figures 1B and C). Furthermore, as shown in Figure 1D, PQ treatment significantly inhibited the cell migration and colony formation in SiHa cervical cancer cells.

$P Q$ treatment accelerates the cellular and mitochondria ROS levels and mitochondrial dysfunction. In order to detect whether PQ can increase intracellular ROS, SiHa cervical cancer cells were treated with PQ in various concentrations $(0,40,60 \mu \mathrm{g} / \mathrm{ml})$ for $24 \mathrm{~h}$ and the cellular ROS levels were detected by DHE \& Hoechst staining. The results showed that PQ can significantly increase the intracellular ROS contents in SiHa cells (Figure 2A). At the same time, MitoSOX (a mitochondiral ROS indicator, which can be oxidized by superoxide in live cells) \& Hoechst staining showed that PQ can also effectively increase ROS levels specifically in mitochondria (Figure 2B). Higher mitochondria ROS levels will cause the disruption of mitochondrial function. In order to determine whether PQinduced ROS leads to oxidative stress that can cause a shift in the redox balance of mitochondria, we examined the increase of mitochondrial permeability in SiHa cells using the permeability of $\mathrm{JC} 1$ as a readout. JC-1 is a membrane permeable dye that selectively enters the mitochondria and can detect the intracellular mitochondrial membrane potential. When the mitochondrial membrane potential is high, JC-1 accumulates inside the mitochondria forming aggregates, which can generate red fluorescence; when the mitochondrial membrane potential is low, JC- 1 cannot be gathered in the matrix of the mitochondria, it remains a monomer and produces green fluorescence (28). The results showed that the ratio of green fluorescence (low $\Delta \Psi \mathrm{m}$ ) to red fluorescence (high $\Delta \Psi \mathrm{m}$ ) of JC-1 in SiHa cells was significantly increased in various concentrations $(0,40,60$ $\mu \mathrm{g} / \mathrm{ml}$ ) of PQ treatment (Figure 2C).
In addition to mitochondrial membrane potential, ATPsynthesis ability is also a fundamental indicator evaluating mitochondrial function (29). Therefore, we examined the intracellular ATP contents of SiHa cells treated with different concentrations $(0,40,60 \mu \mathrm{g} / \mathrm{ml})$ of PQ for $24 \mathrm{~h}$. The results showed that PQ treatment significantly inhibited the ATP synthesis in SiHa cells (Figure 2D).

$P Q$ induces mitochondria-dependent apoptosis and MAPK signaling pathways in SiHa cells. In order to detect whether PQ is responsible for mitochondrial-dependent apoptosis, we treated $\mathrm{SiHa}$ cells with PQ $(60 \mu \mathrm{g} / \mathrm{ml})$ for the indicated duration times $(0,1,3,6,12$ and $24 \mathrm{~h})$, and we then measured the protein levels of different caspases and their upstream B-cell lymphoma-2 (Bcl-2) family proteins. The results showed that the expression levels of cleavedcaspase3, cleaved-caspase 9 and Bax proteins were upregulated in PQ-treated SiHa cells, while the $\mathrm{Bcl}-\mathrm{x}_{\mathrm{L}}$ proteins were down-regulated following PQ treatment (Figure 3A).

MAPK family members, including c-Jun N-terminal kinase (JNK), ERK1/2, and P38 MAPK, are involved in apoptosis (30). Following the same treatment as above, we assessed protein expression level changes of the MAPK signaling pathway. Our results showed that PQ treatment significantly increased the p-P38, p-ERK and p-JNK protein expression levels in SiHa cells (Figure 3B). To understand the role of MAPK in PQ-induced cell apoptosis, the SiHa cells were tested using Annexin-V-FITC/PI staining after they were pretreated with inhibitors of MAPK, such as i) SB203580 (P38 inhibitor, $50 \mathrm{nM}$ ), ii) PD98059 (ERK inhibitor, $10 \mu \mathrm{M})$ and iii) SP600125 (JNK inhibitor, $40 \mathrm{nM}$ ) for $30 \mathrm{~min}$ followed by treatment with PQ for $24 \mathrm{~h}$. The results showed that suppressing P38 with SB203580 significantly decreased the PQ-induced cellular apoptosis in SiHa cells but not ERK or JNK inhibitors (Figures 3C and D).

Inhibition of intracellular ROS reduces mitochondrial damage, cellular apoptosis, cell migration and colony formation in SiHa cells. To detect whether ROS is a key molecule mediating PQ-induced apoptosis, the SiHa cells were pre-treated with N-Acetylcysteine (NAC, ROS inhibitor, $5 \mathrm{mM})$ for $30 \mathrm{~min}$ followed by treatment with PQ $(60 \mu \mathrm{g} / \mathrm{ml})$ for $24 \mathrm{~h}$. The cellular and mitochondrial ROS levels as well as the mitochondrial membrane potential were detected using DHE, MitoSOX and JC-1 staining, respectively, and fluorescence microscopy. The results showed that treatment with NAC significantly reduced the PQ-stimulated cellular and mitochondrial ROS levels as well as the mitochondrial membrane potential in $\mathrm{SiHa}$ cells (Figures 4A-C). Furthermore, as shown in Figure 4D, NAC treatment significantly restored the PQ-induced inhibition of ATP synthesis. We also examined the effect of NAC on PQ with regard to the cell viability, apoptosis, migration, proliferation, 
as well as apoptosis-related protein expression using CCK-8 assay, Annexin-V staining, wound healing assay, colony forming assay and western blot in SiHa cervical cells, respectively. The results showed that scavenging of ROS with NAC treatment significantly restored the PQ-induced increase in cytotoxicity and apoptosis (Figures 5A-C) in SiHa cells, and also recovered the inhibition of cell migration and proliferation (Figure 5D). Western blot analysis showed that NAC could dramatically restore the PQ-induced up-regulation of pro-apoptotic c-cas 3 and c-cas 9 and down-regulation of anti-apoptotic Bcl-xL proteins expression (Figure 5E).

\section{Discussion}

Cancer is a complex process involving a series of proliferative signals, including escape from growth inhibition, resistance to cell death, and uncontrolled proliferation as well as increased invasion and metastasis of cancer cells. A great focus has been on the development of new anti-tumor drugs, including natural plant drugs and their derivatives $(6,31)$. In fact, herbal medicinal have been tested as potential treatment modalities for a variety of cancers, including leukemia, ovarian, testicular, lung, liver, esophageal, stomach, colon, and rectum cancer (32-34). In the present study, we reported that the $P$. quassioides extract bears anti-cancer properties in $\mathrm{SiHa}$ cervical cancer cells with a low cytotoxicity in normal cells, promoting apoptosis and inhibiting cancer cell migration and proliferation.

ROS, known as second messenger, play pivotal roles in cell survival, proliferation, migration and apoptosis (35). It was well known that mitochondria are not only the main source of intracellular ROS production but are considered as the main targets of ROS damage $(36,37)$. In pathological conditions, excessive ROS formation in mitochondria can reduce energy synthesis, such as in the form of ATP, and accelerate the production of additional $\operatorname{ROS}(25,38)$, accumulation of which can damage the mitochondrial membrane and potentially lead to mitochondria-dependent apoptosis in cells $(20,39,40)$.

In this study, our experimental data revealed that PQ treatment significantly increased intracellular and mitochondrial ROS production, as well as ATP synthesis accompanied by a decrease in the mitochondrial membrane potential in $\mathrm{SiHa}$ cervical cancer cells. Members of the caspase and Bcl-2 families are involved in promoting mitochondria-dependent cell apoptosis $(41,42)$. In our research, we found that PQ treatment significantly upregulated the pro-apoptotic proteins, such as cleaved-caspase9, cleaved-caspase 3 and Bax expression levels in SiHa cervical cancer cells while down-regulated the anti-apoptotic protein, Bcl- $\mathrm{x}_{\mathrm{L}}$. Furthermore, ROS inhibition significantly attenuated apoptosis as well as their migration, proliferation and mitochondrial permeability in PQ-treated SiHa cervical cancer cells. All of these findings strongly suggest that ROS is a key point in PQ stimulation of normal function in SiHa cervical cancer cells. Despite, this understanding, the role of PQ on the mitochondrial membrane potential as well as the molecular mechanisms responsible for the increase of cellular ROS levels remains to be addressed in future studies.

Downstream of ROS, the MAPK signaling is involved in many cellular responses, such as senescence, proliferation, differentiation, and apoptosis $(41,43)$. Members of the MAPK signaling involve the P38, ERK and JNK, whose pathways have been linked to apoptosis in various cancer cells as well as in cervical cancer $(44,45)$. Recently, it was reported that a Brevilin A induces apoptosis in Breast Cancer Cells through the activation the JNK and P38/MAPK (46), while inhibition of the ERK-MAPK efficiently prevented apoptosis in hepatocellular carcinoma cells (47). In the present study, we also reported that PQ treatment significantly up-regulated the phosphorylation of P38, suggesting that MAPK signaling is involved in the PQstimulated cell apoptosis in SiHa cervical cancer cells.

There are some limitations in this study. First, the extract from $P$. quassioides is not a compound, thus we can't ensure the correct component that is responsible for activating apoptosis in cells, and second, we have no direct evidence to understand the molecular mechanisms linking ROS, P38/MAPK and mitochondrial dysfunction. Further studies will give answers to these questions.

In conclusion, our results revealed that $P$. quassioides extract exerts anti-cancer properties by increasing cytotoxicity and apoptosis, and inhibiting migration and proliferation in SiHa cervical cancer cells. The disruption of these cellular functions was caused by the accumulation of intracellular and mitochondrial ROS which in turn activated the P38/MAPK and the mitochondria/dependent apoptosis signaling pathway. Our findings provide insight for $P$. quassioides as a potential therapeutic strategy for the treatment of cervical cancer.

\section{Funding}

This research was supported by the project for postgraduate to innovate scientific research in Heilongjiang Bayi Agricultural University (No: YJSCX2019-Y64), the Natural Science Foundation of Heilongjiang Province of China (QC2015121), and the scientific research team support plan of Heilongjiang Bayi Agricultural University (TDJH201904), China.

\section{Conflicts of Interest}

The Authors declare that they have no competing interests.

\section{Authors' Contributions}

YXG, YL, YHJ, TK, YDC and HNS performed the construct of the model and wrote the manuscript. YHH, JL, GNS, DPX, CXR, LYY, 
DSL, JSK, YJJ, and JK performed the data analysis, JL and YHP provided $P$. quassioides extract reagents, TK, YDC and HNS were substantial contributors to study conception and design. All authors read and approved the final manuscript.

\section{Acknowledgements}

This research was supported by Basic Science Research Program through the National Research Foundation of Korea (NRF) funded by the Ministry of Education (2017R1D1A1B03028188). This study was supported by grants from the Korean Research Institute of Bioscience and Biotechnology Research Initiative Program (KRIBB) (KGM5162021).

\section{References}

1 Small Jr W, Bacon MA, Bajaj A, Chuang LT, Fisher BJ, Harkenrider MM, Jhingran A, Kitchener HC, Mileshkin LR and Viswanathan AN: Cervical cancer: A global health crisis. Cancer 123(13): 24042412, 2017. PMID: 28464289. DOI: 10.1002/cncr.30667

2 Kumar L, Harish P, Malik PS and Khurana S: Chemotherapy and targeted therapy in the management of cervical cancer. Curr Probl Cancer 42(2): 120-128, 2018. PMID: 29530393. DOI: 10.1016/j.currproblcancer.2018.01.016

3 Cohen PA, Jhingran A, Oaknin A and Denny L: Cervical Cancer. Lancet 393 (10167), 169-182, 2019. PMID: 30638582. DOI: 10.1016/S0140-6736(18)32470-X

4 Elkady AI: Crude alkaloid extract of rhazya stricta inhibits cell growth and sensitizes human lung cancer cells to cisplatin through induction of apoptosis. Genet Mol Biol 36(1): 12-21, 2013. PMID: 23569403. DOI: $10.1590 / \mathrm{S} 1415-47572013005000009$

5 Chandimali N, Sun HN, Kong LZ, Zhen X, Liu R, Kwon T and Lee DS: Shikonin-induced apoptosis of colon cancer cells is reduced by peroxiredoxin V expression. Anticancer Res 39(11): 6115-6123, 2019. PMID: 31704839. DOI: 10.21873/anticanres.13819

6 Evans W, Filippova M, Filippov V, Bashkirova S, Zhang G, Reeves ME and Duerksen-Hughes P: The ethanol crude extraction of cyperus rotundus regulates apoptosis-associated gene expression in HeLa human cervical carcinoma cells in vitro. Anticancer Res 39(7): 3697-3709, 2019. PMID: 27365376. DOI: 10.21873 /anticanres.13518

7 Fan H, Qi D, Yang M, Fang H, Liu K and Zhao F: In vitro and in vivo anti-inflammatory effects of 4-methoxy-5hydroxycanthin-6-one, a natural alkaloid from Picrasma quassioides. Phytomedicine 20(3-4): 319-323, 2013.PMID: 23271002. DOI: 10.1016/j.phymed.2012.11.016

8 Jiao W-H, Gao H, Zhao F, Lin H-W, Pan Y-M, Zhou G-X and Yao X-S: Anti-inflammatory alkaloids from the stems of Picrasma quassioides BENNET. Chem Pharm Bull 59(3): 359364, 2011. PMID: 21372418. DOI: 10.1248/cpb.59.359

9 Nurhanan M, Hawariah LA, Ilham AM and Shukri MM: Cytotoxic effects of the root extracts of eurycoma longifolia jack. Phytotherapy Res 19(11): 994-996, 2005. PMID: 16317660. DOI: $10.1002 /$ ptr.1759

10 Zhao W, Yu J, Su Q, Liang J, Zhao L, Zhang Y and Sun W: Antihypertensive effects of extract from picrasma quassiodes (d. Don) benn. In spontaneously hypertensive rats. J Ethnopharmacol 145(1): 187-192, 2013. PMID: 23142488. DOI: 10.1016/j.jep.2012.10.049
11 Tyacke R, Lau A, Grella B, Glennon R, Nutt D and Hudson A: Investigation of the affinities of two new $\mathrm{b}$-carbolines for rat brain imidazoline2 receptors. Ann N Y Acad Sci 1009(1): 361363, 2003. PMID: 15028612. DOI: 10.1196/annals.1304.047

12 Zhao W-Y, Zhou W-Y, Chen J-J, Yao G-D, Lin B, Wang X-B, Huang $\mathrm{X}-\mathrm{X}$ and Song S-J: Enantiomeric $\beta$-carboline dimers from picrasma quassioides and their anti-hepatoma potential. Phytochemistry 159(3): 39-45, 2019. PMID: 30577000. DOI: 10.1016/j.phytochem.2018.12.002

13 Dejos C, Voisin P, Bernard M, Régnacq M and Bergès T: Canthin6-one displays antiproliferative activity and causes accumulation of cancer cells in the G2/M phase. J Nat Prod 77(11): 2481-2487, 2014. PMID: 25379743. DOI: 10.1021/np500516v

14 Jiang M-X and Zhou Y-J: Canthin-6-one alkaloids from picrasma quassioides and their cytotoxic activity. J Asian Nat Prod Res 10(11-12): 1009-1012, 2008. PMID: 19031238. DOI: 10.1080/ 10286020802277956

15 Zhao W-Y, Chen J-J, Zou C-X, Zhou W-Y, Yao G-D, Wang XB, Lin B, Huang X-X and Song S-J: Effects of enantiomerically pure $\beta$-carboline alkaloids from picrasma quassioides on human hepatoma cells. Planta Med 85(08): 648-656, 2019. PMID: 30974464. DOI: 10.1055/a-0879-4721

16 Lou L L, Yao G D , Wang J, Zhao WY, Wang XB, Huang XX and Song SJ: Enantiomeric neolignans from, Picrasma quassioides, exhibit distinctive cytotoxicity on hepatic carcinoma cells through ROS generation and apoptosis induction. Bioorg Med Chem Lett 28(8): 1263-1268, 2018 PMID: 29567344. DOI: 10.1016/j.bmcl.2018.03.043

17 Yao GD, Wang J, Song XY, Zhou L, Lou LL, Zhao WY, Lin B, Huang XX and Song SJ: Stereoisomeric guaiacylglycerol- $\beta$ coniferyl aldehyde ether induces distinctive apoptosis by downregulation of MEK/ERK pathway in hepatocellular carcinoma cells. Bioorg Chem 81(12): 382-388, 2018. PMID: 30196208. DOI: $10.1016 /$ j.bioorg.2018.08.033

18 Angelova PR and Abramov AY: Functional role of mitochondrial reactive oxygen species in physiology. Free Radic Biol Med 100(11): 81-85, 2016. PMID: 27296839. DOI: 10.1016/j. freeradbiomed.2016.06.005

19 Sena LA and Chandel NS: Physiological roles of mitochondrial reactive oxygen species. Mol Cell 48(2): 158-167, 2012. PMID: 23102266. DOI: 10.1016/j.molcel.2012.09.025

20 Moloney JN and Cotter TG: ROS signalling in the biology of cancer. Semin Cell Dev Biol 80(8): 50-64, 2018. PMID: 28587975. DOI: $10.1016 /$ j.semcdb.2017.05.023

21 Chio IIC and Tuveson DA: ROS in cancer: The burning question. Trends Mol Med 23(5): 411-429, 2017. PMID: 28427863. DOI: 10.1016/j.molmed.2017.03.004

22 Martin K and Barrett J: Reactive oxygen species as doubleedged swords in cellular processes: Low-dose cell signaling versus high-dose toxicity. Hum Exp Toxicol 21(2): 71-75, 2002. PMID: 12102499. DOI: 10.1191/0960327102ht213oa

23 Schumacker PT: Reactive oxygen species in cancer: A dance with the devil. Cancer Cell 27(2): 156-157, 2015. PMID: 25670075. DOI: 10.1016/j.ccell.2015.01.007

24 Zorov DB, Juhaszova M and Sollott SJ: Mitochondrial reactive oxygen species (ros) and ros-induced ros release. Physiol Rev 94(3): 909-950, 2014. PMID: 24987008. DOI: 10.1152/ physrev.00026.2013

25 Sabharwal SS and Schumacker PT: Mitochondrial ROS in cancer: initiators, amplifiers or an Achilles' heel? Nat Rev 
Cancer 14(11): 709-721, 2014. PMID: 25342630. DOI: 10.1038/ nrc3803

26 Pfeffer CM and Singh AT: Apoptosis: A target for anticancer therapy. Int J Mol Sci. 19(2): 448, 2018. PMID: 29393886. DOI: 10.3390/ijms 19020448

27 Bernardi P, Rasola A, Forte M and Lippe G: The mitochondrial permeability transition pore: Channel formation by F-ATP synthase, integration in signal transduction, and role in pathophysiology. Physiol Rev 95(4): 1111-1155, 2015. PMID: 26269524. DOI: 10.1152/physrev.00001.2015

28 Mo XQ, Wei HY, Huang GR, Xu LY, Chen YL, Qi J, Xian W, Qin YC, Wei LD, Zhao LJ, Huang YQ, Xing W, Pu HQ, Wei PY, Li CG and Liang QC: Molecular mechanisms of apoptosis in hepatocellular carcinoma cells induced by ethanol extracts of Solanum lyratum Thumb through the mitochondrial pathway. World J Gastroenterol 23(06): 1010-1017, 2017. PMID: 28246474. doi:10.3748/wjg.v23.i6.1010

29 Xu MF, Xiong YY, Liu JK, Qian JJ, Zhu L and Gao J: Asiatic acid, a pentacyclic triterpene in Centella asiatica, attenuates glutamate-induced cognitive deficits in mice and apoptosis in SH-SY5Y cells. Acta Pharmacol Sin 33(5): 578-587, 2012. PMID: 22447225. DOI: 10.1038/aps.2012.3

30 Bagkos G, Koufopoulos K and Piperi C: ATP synthesis revisited: new avenues for the management of mitochondrial diseases. Curr Pharm 20(28): 4570-4579, 2014. PMID: 24372303. DOI: $10.2174 / 1381612819666131230124334$

31 Boldt S, Weidle UH and Kolch W: The role of MAPK pathways in the action of chemotherapeutic drugs. Carcinogenesis 23(11): 1831-1838, 2002. PMID: 12419831. DOI: 10.1093/carcin/ 23.11.1831

32 Hadi S, Asad S, Singh S and Ahmad A: Putative mechanism for anticancer and apoptosis-inducing properties of plant-derived polyphenolic compounds. IUBMB Life 50(3): 167-171, 2000. PMID: 11142343. DOI: 10.1080/152165400300001471

33 Ying J, Zhang M, Qiu X and Lu Y: The potential of herb medicines in the treatment of esophageal cancer. Biomed Pharmacother 103(7): 381-390, 2018. PMID: 29674273. DOI: 10.1016/j.biopha.2018.04.088

34 Hu LL, Chen DS, Wang YY, Qin Y, Huang P, Yu LX, Liao J and Hua XL: Smilax China L. rhizome extract inhibits nuclear factor- $\mathrm{kB}$ and induces apoptosis in ovarian cancer cells. Chin J Integr Med 21(12): 907-915, 2015. PMID: 24411428. DOI: $10.1007 / \mathrm{s} 11655-014-1788-9$

35 Lin L-J and Huang HY: DFSG, a novel herbal cocktail with antiasthma activity, suppressed MUC5AC in A549 cells and alleviated allergic airway hypersensitivity and inflammatory cell infiltration in a chronic asthma mouse model. Biomed Pharmacother 121(1): 109584, 2020. PMID: 31766098. DOI: 10.1016/j.biopha.2019.109584

36 Liochev SI: Reactive oxygen species and the free radical theory of aging. Free Radic Biol Med 60(7): 1-4, 2013. PMID: 23434764. DOI: 10.1016/j.freeradbiomed.2013.02.011

37 Ott M, Gogvadze V, Orrenius S and Zhivotovsky B: Mitochondria, oxidative stress and cell death. Apoptosis 12(5): 913-922, 2007. PMID: 17453160. DOI: 10.1007/s10495-0070756-2
38 Crack PJ and Taylor JM: Reactive oxygen species and the modulation of stroke. Free Radic Biol Med 38(11): 1433-1444, 2005. PMID: 15890617. DOI: 10.1016/j.freeradbiomed. 2005.01.019

39 Pelicano H, Carney D and Huang P: Ros stress in cancer cells and therapeutic implications. Drug Resist Updat 7(2): 97-110, 2004. PMID: 15158766. DOI: 10.1016/j.drup.2004.01.004

40 Ryu SY, Peixoto PM, Teijido O, Dejean LM and Kinnally KW: Role of mitochondrial ion channels in cell death. Biofactors 36(4): 255-263, 2010. PMID: 20623547. DOI: 10.1002/biof.101

41 Evans W, Filippova M, Filippov V, Bashkirova S, Zhang G, Reeves ME and Duerksen-Hughes P: Overexpression of HPV16 E6* alters $\beta$-integrin and mitochondrial dysfunction pathways in cervical cancer cells. Cancer Genomics Proteomics 13(4): 259273, 2016. PMID: 27365376.

42 Kim HJ, Park C, Han MH, Hong SH, Kim GY, Hoon Hong S, Deuk Kim N and Choi YH: Baicalein induces caspase-dependent apoptosis associated with the generation of ROS and the activation of AMPK in human lung carcinoma A549 Cells. Drug Dev Res 77(2): 73-86, 2016. PMID: 26971531. DOI: $10.1002 / \mathrm{ddr} .21298$

43 Zhao X, Tao X, Xu L, Yin L, Qi Y, Xu Y, Han X and Peng J: Dioscin induces apoptosis in human cervical carcinoma HeLa and SiHa cells through ROS-mediated DNA damage and the mitochondrial signaling pathway. Molecules 21(6): 730, 2016. PMID: 27271587. DOI: 10.3390/molecules21060730

44 Zhang Z, Wang L, Du J, Li Y, Yang H, Li C, Li H and Hu H: Lipid raft localization of epidermal growth factor receptor alters matrix metalloproteinase-1 expression in SiHa cells via the MAPK/ERK signaling pathway. Oncol Lett 12(6): 4991-4998, 2016. PMID: 28101233. DOI: 10.3892/ol.2016.5307

45 Xia X, Xiang X, Huang F, Zheng M, Zhang Z and Han L: Dietary canolol induces apoptosis in human cervical carcinoma HeLa cells through ROS-MAPK mediated mitochondrial signaling pathway: in vitro and in vivo. Chem Biol Interact 300: 138-150, 2019. PMID: 30653946. DOI: 10.1016/j.cbi. 2019.01.016

46 Schumacker PT: Reactive oxygen species in cancer cells: live by the sword, die by the sword. Cancer Cell 10(3): 175-176, 2006. PMID: 16959608. DOI: 10.1016/j.ccr.2006.08.015

47 Saleem MZ, Nisar MA, Alshwmi M, Din SRU, Gamallat Y, Khan M and Ma T: Brevilin A inhibits STAT3 signaling and induces ROS-dependent apoptosis, mitochondrial stress and endoplasmic reticulum stress in MCF-7 breast cancer cells. Onco Targets Ther 1(13): 435-450, 2020. PMID: 32021288. DOI: 10.2147/OTT.S228702

48 Lin-Yen K, Chen WL, Chen JH, Hsu FT, Liu TT, Chen WT, Wang KL, Chen WC, Liu YC and Wang WS: Magnolol induces apoptosis and inhibits ERK-modulated metastatic potential in hepatocellular carcinoma cells. In Vivo 32(6): 1361-1368, 2018. PMID: 30348689. DOI: 10.21873/invivo.11387

Received March 10, 2020

Revised March 24, 2020

Accepted April 2, 2020 